\title{
ACHADOS ULTRA-SONOGRÁFICOS ABDOMINAIS EM PACIENTES COM DENGUE*
}

\author{
Karen Amaral do Vabo ${ }^{1}$, Gilberto Torres Neto ${ }^{2}$, Alair Augusto S.M.D. dos Santos ${ }^{3}$, \\ Telmo Pimentel do Vabo $^{4}$, Maria Lúcia de Oliveira Santos ${ }^{4}$, Edson Marchiori ${ }^{5}$
}

Resumo OBJETIVO: Apresentar os achados ultra-sonográficos abdominais em pacientes com dengue e compará-los aos descritos na literatura. MATERIAIS E MÉTODOS: Foram realizados exames ultra-sonográficos abdominais de 38 pacientes, 25 do sexo feminino e 13 do sexo masculino, com idade média de 35 anos, com diagnóstico de dengue sorologicamente confirmado. Os achados foram comparados com os descritos na literatura. RESULTADOS: Os achados ultra-sonográficos mais relevantes foram espessamento difuso da parede da vesícula biliar em 18 casos $(47,4 \%)$, líquido livre na cavidade abdominal e/ou pélvica em 12 (31,6\%), esplenomegalia em 11 (28,9\%), hepatomegalia em 10 (26,3\%) e líquido pericolecístico em 10 (26,3\%). Vinte e seis por cento dos pacientes apresentaram exames ultra-sonográficos normais. CONCLUSÃO: Os achados ultra-sonográficos abdominais são uma ferramenta adicional útil na confirmação de casos suspeitos de dengue hemorrágica e na detecção precoce da gravidade e da progressão da doença, sendo de extrema importância para o radiologista o conhecimento destes possíveis achados.

Unitermos: Dengue; Ultra-sonografia; Manifestações abdominais.

Abstract Abdominal ultrasound findings in patients with dengue fever.

OBJECTIVE: To review the abdominal ultrasound findings in patients with serologically proven dengue fever and to compare the results with data from the literature. MATERIALS AND METHODS: Thirty-eight patients with serologically proven dengue fever, 25 female and 13 male, mean age of 35 years, were submitted to abdominal ultrasound. The ultrasound findings were compared with data from the literature. RESULTS: The most relevant ultrasound findings were diffuse gallbladder wall thickening in 18 cases $(47.4 \%)$, abdominal and/or pelvic free fluid in $12(31.6 \%)$, splenomegaly in $11(28.9 \%)$, hepatomegaly in $10(26.3 \%)$ and perivesicular fluid in $10(26.3 \%)$. Twenty-six percent of the patients had normal abdominal ultrasound. CONCLUSION: Abdominal sonography is a useful additional diagnostic tool for the confirmation of suspected cases of dengue hemorrhagic fever and for the evaluation of severity and progression of the disease. The knowledge of the ultrasound possible findings in patients with dengue fever is of extreme importance for the radiologist. Key words: Dengue; Ultrasound; Abdominal findings.

\section{INTRODUÇÃO}

O dengue é uma doença febril aguda, cujo agente etiológico é um vírus do gênero flavivírus $^{(1)}$. É uma arbovirose transmitida ao homem pela picada do mosquito Aedes

* Trabalho realizado no Setor de Imagens do Hospital de Clínicas de Niterói (HCN) e no Departamento de Radiologia do Hospital Universitário Antônio Pedro (HUAP) da Universidade Federal Fluminense (UFF), Niterói, RJ.

1. Médica Pós-graduanda de Radiologia da UFF.

2. Professor do Departamento de Radiologia da Universidade do Estado do Rio de Janeiro (UERJ), Médico do Setor de Imagens do $\mathrm{HCN}$.

3. Professor Adjunto do Departamento de Radiologia da UFF Chefe do Serviço de Radiologia do HUAP-UFF, Coordenador do Setor de Imagens do HCN.

4. Professores Adjuntos do Departamento de Radiologia da UFF, Médicos do Setor de Imagens do HCN.

5. Professor Titular de Radiologia da UFF, Coordenador Ad junto do Curso de Pós-Graduação em Radiologia da Universidade Federal do Rio de Janeiro (UFRJ).

Endereço para correspondência: Prof. Dr. Edson Marchiori. Rua Thomaz Cameron, 438, Valparaíso. Petrópolis, RJ, 25685-120. E-mail: edmarchiori@bol.com.br

Recebido para publicação em 12/8/2003. Aceito, após revisão, em 2/9/2003. aegypti $^{(\mathbf{1})}$. São conhecidos, atualmente, quatro sorotipos virais: DEN-1, DEN-2, DEN-3 e DEN-4 ${ }^{(\mathbf{1 , 2})}$. As formas clinicamente mais graves parecem estar relacionadas às infecções subseqüentes por diferentes sorotipos ${ }^{(3-5)}$.

No Brasil, desde 1986, ocorrem epidemias anuais de dengue, até então pelos sorotipos 1 e 2 , tornando-se um problema nacional de saúde pública ${ }^{(\mathbf{5})}$.

O estado do Rio de Janeiro viveu, em 2002, a maior epidemia de dengue já descrita, com a introdução de um novo sorotipo (sorotipo 3), isolado pela primeira vez no estado em janeiro de $2001^{(\boldsymbol{6})}$. A introdução de um novo sorotipo em uma população suscetível propiciou o aparecimento de formas mais graves da doença ${ }^{(5,7)}$.

A Organização Mundial da Saúde (OMS) classifica o dengue em duas formas clínicas: o dengue clássico, em que os principais sintomas são febre alta, cefaléia, dor retroorbitária, mialgia, artralgia, exantema, dor abdominal e fenômenos hemorrágicos leves; e a febre hemorrágica do dengue, que se inicia como o dengue clássico, porém evolui rapidamente para manifestações hemorrágicas, derrames cavitários, instabilidade hemodinâmica e/ou choque ${ }^{(7)}$.

A ultra-sonografia tem sido consideravelmente utilizada na avaliação da dor abdominal em quadros febris agudos. Uma diversidade de achados ultra-sonográficos abdominais é descrita no dengue, sendo o seu conhecimento relevante para reforçar a hipótese em casos suspeitos e excluir possíveis diagnósticos diferenciais ${ }^{(\mathbf{8}-10)}$.

No presente trabalho são descritas as alterações ultra-sonográficas abdominais encontradas em pacientes internados no Hospital de Clínicas de Niterói (HCN), RJ, no período de janeiro a junho de 2002, com diagnóstico de dengue sorologicamente confirmado. 


\section{MATERIAIS E MÉTODOS}

No período de janeiro a junho de 2002 , 38 pacientes adultos com diagnóstico de dengue sorologicamente confirmado (teste Elisa), sendo 25 mulheres e 13 homens, com idades entre 16 e 60 anos, média de 35 anos, foram submetidos a exames ultrasonográficos abdominais no HCN.

Todos os exames foram realizados em aparelho Hewlett Packard - Image Point HX, com sonda de 3,5-5 ou 7,5-10 MHz.

Os exames ultra-sonográficos abdominais eram solicitados para pacientes apresentando quadro clínico sugestivo de dengue, com dor abdominal de forte intensidade, para melhor avaliação da gravidade do quadro, além de afastar possíveis diagnósticos diferenciais. Foram procurados sinais de hepatomegalia, esplenomegalia, líquido livre na cavidade abdominal e/ou pélvica e espessamento da parede da vesícula biliar.

Considerou-se hepatomegalia quando o comprimento hepático mediu mais que 155 mm na linha hepática média, e esplenomegalia quando o baço tinha comprimento maior que $120 \mathrm{~mm}^{(\mathbf{1 1})}$. O espessamento da parede da vesícula biliar foi considerado quando a medida da espessura ultrapassava $3 \mathrm{~mm}^{(8,10,11)}$.

\section{RESULTADOS}

Dos 38 pacientes estudados, 10 (26,3\%) apresentavam exames ultra-sonográficos normais. Os demais 28 pacientes $(73,6 \%)$ apresentavam alterações que incluíam: espessamento difuso da parede da vesícula biliar em 18 (47,4\%), estando associado a líquido pericolecístico em 10 (26,3\%); líquido livre em cavidade abdominal ou pélvica em 12 (31,6\%); esplenomegalia em 11 (28,9\%); hepatomegalia em 10 (26,3\%). Outros achados menos freqüentes foram vesícula biliar hiperdistendida (diâmetro transverso da vesícula maior que $5 \mathrm{~cm}$ ) em dois $(5,3 \%)$ pacientes, derrame pleural em um $(2,6 \%)$ e dilatação da via biliar intrahepática em um (2,6\%) (Tabela 1).

$\mathrm{O}$ espessamento difuso da parede da vesícula biliar (> $3 \mathrm{~mm}$ ) foi o achado mais frequiente. A espessura da parede deste órgão variou entre 3,9 e 11,2 mm. Além do espessamento da parede da vesícula biliar,
Tabela 1 Achados ultra-sonográficos abdominais em pacientes com dengue.

\begin{tabular}{|l|r|r|}
\hline \multicolumn{1}{|c|}{ Achados ultra-sonográficos } & $\mathrm{N}$ & \multicolumn{1}{c|}{$\%$} \\
\hline Espessamento da parede da vesícula & & \\
biliar & 18 & 47,4 \\
Líquido livre na cavidade abdominal & 12 & 31,6 \\
Esplenomegalia & 11 & 28,9 \\
Hepatomegalia & 10 & 26,3 \\
Líquido pericolecístico & 10 & 26,3 \\
Vesícula hiperdistendida & 2 & 5,3 \\
Derrame pleural & 1 & 2,6 \\
Dilatação da via biliar intra-hepática & 1 & 2,6 \\
\hline
\end{tabular}

havia também líquido pericolecístico em $26,3 \%$ dos casos (Figura 1).

A esplenomegalia (>120 $\mathrm{mm}$ ) era homogênea em todos os casos, com medidas variando entre 130 e $150 \mathrm{~mm}$ (Figura 2). A hepatomegalia também era homogênea, sem evidência de lesões focais.

Diferentes volumes de líquido livre, com características de transudato, sem septos ou "débris", foram encontrados na cavidade abdominal e/ou pélvica (Figura 3).

$\mathrm{O}$ derrame pleural observado em um paciente era bilateral e de pequena monta.

\section{Considerações clínicas especiais}

Quatro pacientes, todas do sexo feminino, idade média de 41 anos, com febre hemorrágica do dengue, apresentaram quadro clínico e laboratorial compatível com colecistite alitiásica, com dor intensa no hipocôndrio direito e especificamente no ponto cístico, febre, náuseas e vômitos.

Aos exames ultra-sonográficos abdominais havia espessamento da parede da vesícula biliar, líquido entre seus folhetos parietais e pericolecístico em todos os casos. Ascite foi observada em três casos, derrame pleural bilateral em um e esplenomegalia em um (Figura 4). O tratamento instituído nestes casos foi conservador e de suporte, com evolução satisfatória em todos.

\section{DISCUSSÃO}

O principal fator fisiopatológico da febre hemorrágica do dengue é um importante aumento da permeabilidade vascular, levando a uma perda de plasma e albumina a partir do espaço intravascular, determinando um quadro descrito como polisserosite $^{(3,8)}$. Analisando autópsias de 100 pacientes com febre hemorrágica do dengue, Bhamarapravati et al. ${ }^{(\mathbf{1 2})}$ encontraram efusão serosa em cavidades pleural, peritoneal e pericárdica e edema das membranas serosas da parede da vesícula biliar na maioria dos pacientes.

O diagnóstico e tratamento precoce podem amenizar ou prevenir as complicações, como hemorragia, hipovolemia e choque $^{(7,8)}$. A ultra-sonografia, como modalidade diagnóstica, pode ser uma ferramenta a mais na diferenciação dos casos leves daqueles com maior risco de evolução para a febre hemorrágica do dengue, e no reconhecimento de casos atípicos ${ }^{(8)}$.

Os achados ultra-sonográficos do dengue têm sido mais descritos em populações pediátricas $^{(\mathbf{8}, 10,13)}$. Thulkar et al. ${ }^{(14)}$, em estudo realizado em adultos, consideram que os achados ultra-sonográficos encontrados são similares aos descritos em crianças, embora as anormalidades possam ser menos significativas em adultos.

Espessamento difuso da parede da vesícula biliar é um achado inespecífico, não necessariamente relacionado a uma afecção primária da vesícula ${ }^{(\mathbf{1 0})}$. Esse aspecto foi descrito pela primeira vez em crianças com febre hemorrágica do dengue por Pramuljo e Harun ${ }^{(10)}$, em 1991. Posteriormente, Setiawan et al. ${ }^{(\mathbf{1 3})}$, também em crianças, demonstraram que o espessamento da parede da vesícula, acima de $3 \mathrm{~mm}$, estava significativamente associado a casos mais graves da doença, podendo ser usado como critério para admissão hospitalar e monitoração, e uma espessura maior que $5 \mathrm{~mm}$ identificava pacientes com febre hemorrágica do dengue, com maior risco de desenvolvimento de choque hipovolêmico.

Hepatomegalia esteve presente em $30 \%$ dos nossos casos. Este achado é freqüente, segundo a literatura consultada, e a hepatomegalia dolorosa é um dos indicadores de gravidade. Em autópsias, Bhamarapravati et al. ${ }^{(\mathbf{1 2})}$ encontraram fígado aumentado em 58\% dos casos, com alterações gordurosas, necrose focal e hemorragia demonstráveis na histologia.

O aumento esplênico já havia sido descrito, não só em estudos sonográficos ${ }^{(\mathbf{1 0 , 1 4})}$, mas também como achado de autópsias ${ }^{(\mathbf{1 2})}$.

Achados de ascite e derrame pleural são classicamente descritos na febre hemorrágica do dengue, tanto em crianças como em 

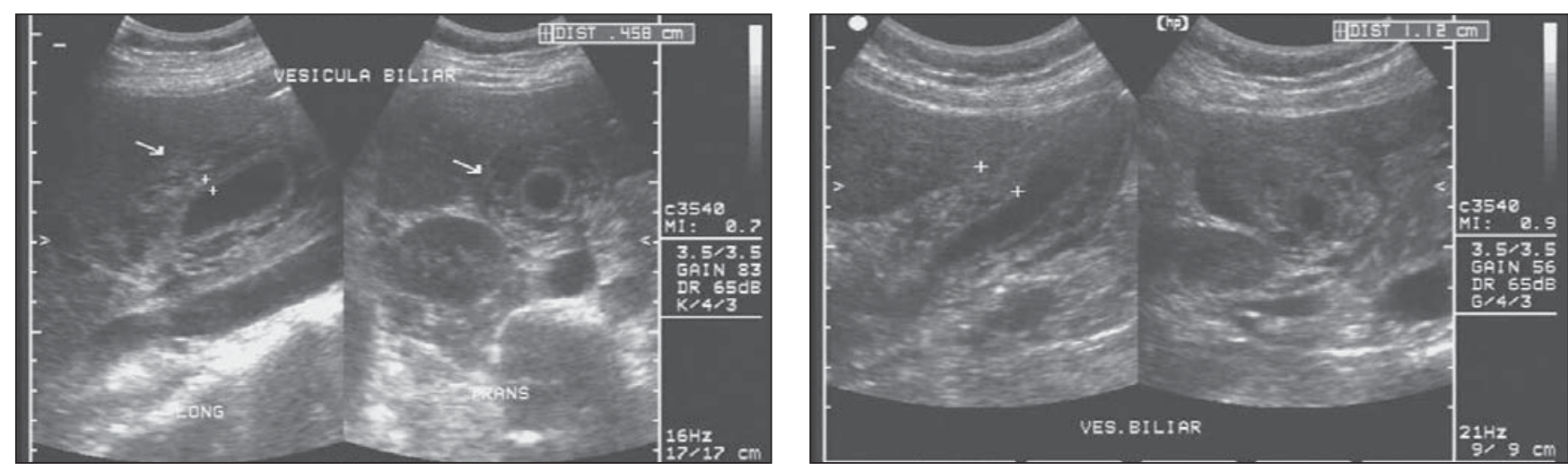

\section{A}

Figura 1. Espessamento da parede da vesícula biliar. Vesícula biliar de parede levemente espessada, medindo 4,5 $\mathrm{mm}$ (A). Grande espessamento da parede da vesícula biliar, com líquido entre seus folhetos parietais e pericolecístico (B). Líquido pericolecístico (C,D).
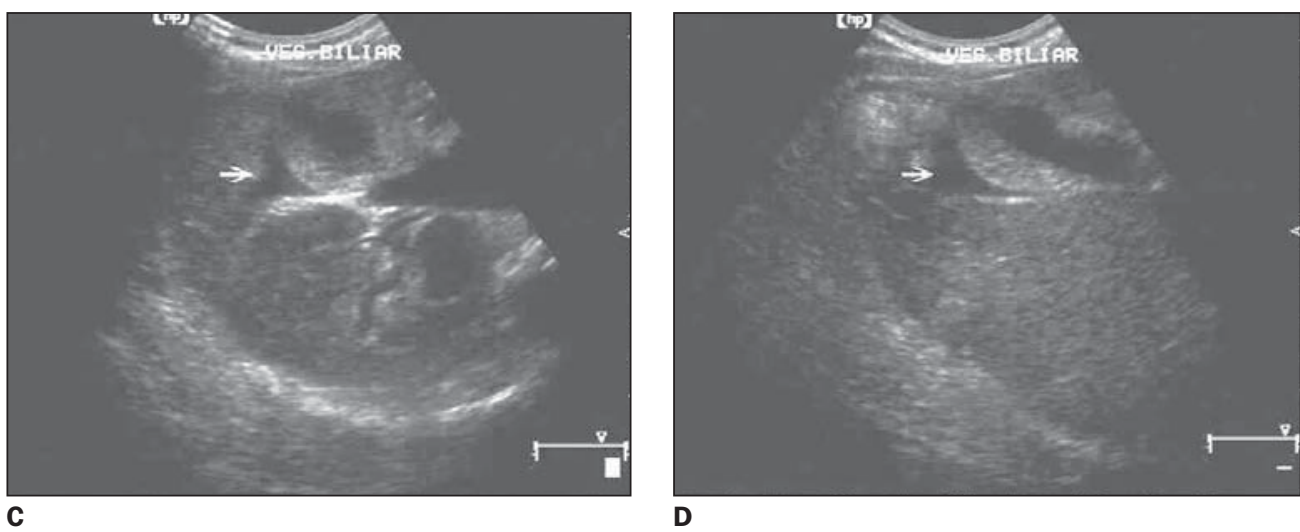

D

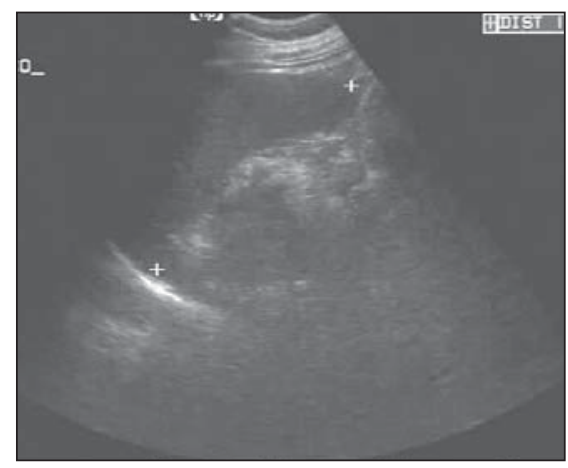

Figura 2. Esplenomegalia homogênea. 0 baço mede $145 \mathrm{~mm}$ de diâmetro longitudinal. adultos, e justificados pelo aspecto fisiopatológico de polisserosite. Existe correlação significativa entre esses achados e a gravidade da doença $\mathrm{a}^{(3,8,10,13)}$.

Derrame pleural é achado freqüente em pacientes com dengue, ocorrendo com mais freqüência bilateralmente, ou apenas à direita ${ }^{(\mathbf{8}, 10)}$. São raros os casos relatados de derrame pleural somente à esquerda ${ }^{(3)}$. Em nosso estudo, derrame pleural bilateral foi evidenciado em apenas um caso.

Um caso de colecistite alitiásica aguda associada à febre hemorrágica do dengue já havia sido descrito por Van Troys et $a l .{ }^{(15)}$, em 2000, sendo considerado uma forma grave e ameaçadora do dengue, de mecanismo fisiopatológico ainda não esclarecido.

Estudo realizado por Setiawan et al. ${ }^{(\mathbf{1 6})}$, em população pediátrica com febre hemorrágica do dengue e queixa de dor epigástrica, evidenciou alargamento difuso do pâncreas em $29 \%$ dos pacientes, sendo este achado mais freqüente nos casos mais graves da doença, podendo estar associado a um aumento nos níveis séricos de amilase

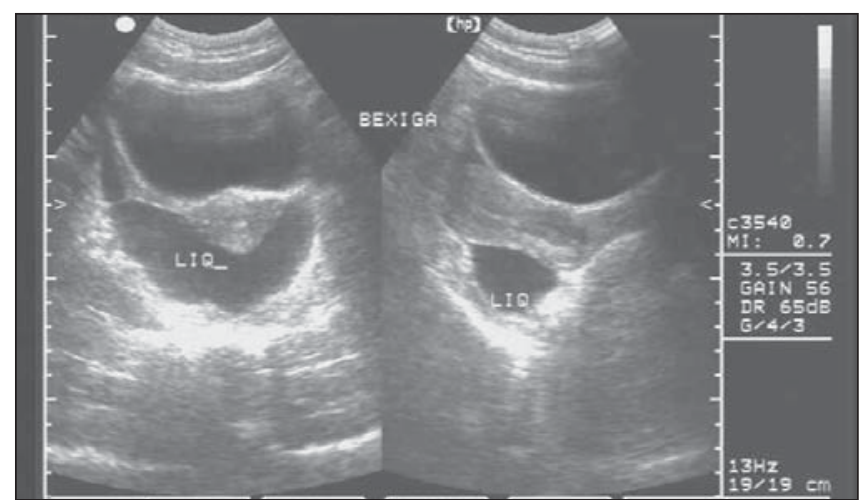

A

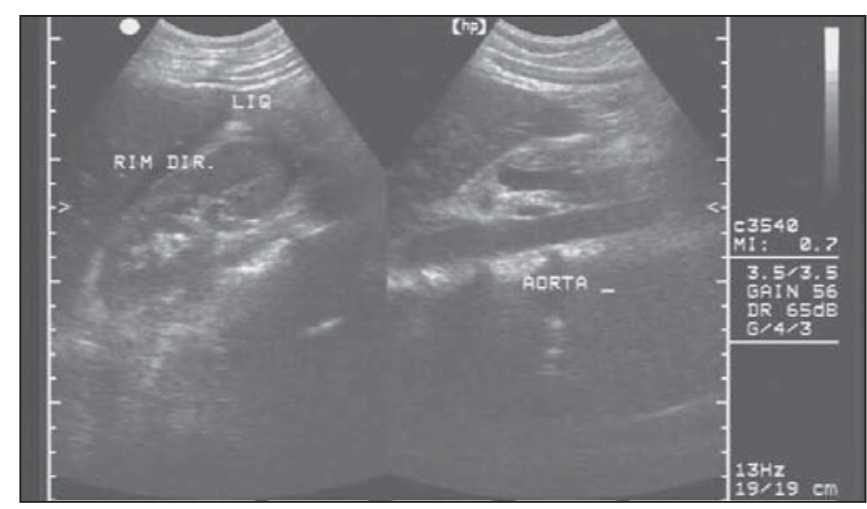

B

Figura 3. Líquido livre na cavidade pélvica de paciente de 19 anos de idade, sem hidratação venosa prévia (A). Líquido no espaço hepatorrenal (B). 


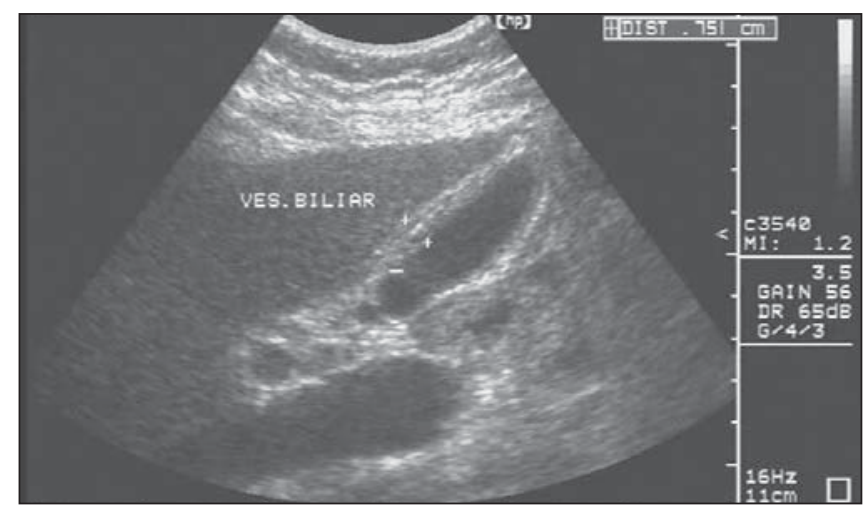

A

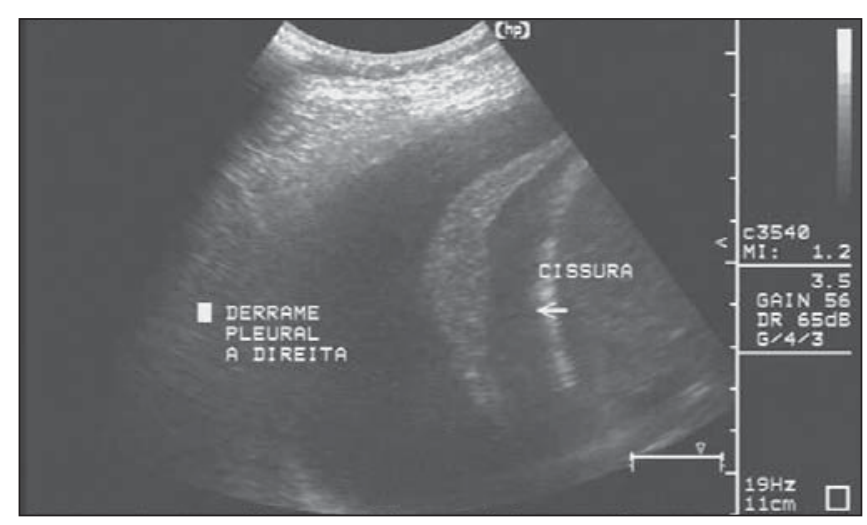

C

Figura 4. Colecistite alitiásica. Paciente de 51 anos de idade apresentando quadro clínico de colecistite alitiásica, evidenciando-se vesícula biliar de paredes espessadas (A), líquido livre na cavidade abdominal (B) e derrame pleural bilateral (C,D).

e lipase. Não encontramos alterações pancreáticas nos pacientes do nosso estudo.

Em resumo, a ultra-sonografia mostrouse um método capaz de detectar as alterações abdominais provocadas pelo dengue. Os achados mais encontrados foram espessamento difuso da parede da vesícula biliar $(47,4 \%)$, líquido livre na cavidade abdominal ou pélvica $(31,6 \%)$, esplenomegalia $(28,9 \%)$ e hepatomegalia $(26,3 \%)$.

Entre as manifestações abdominais atípicas, encontrou-se colecistite alitiásica em quatro casos.

O conhecimento dos possíveis achados ultra-sonográficos abdominais em pacientes com dengue é de extrema relevância para o ultra-sonografista. Esta modalidade diagnóstica pode ser uma importante ferramenta adicional na confirmação de casos suspeitos de febre hemorrágica do dengue e na identificação precoce de casos graves ou passíveis de complicações, além de auxiliar no diagnóstico diferencial de outros quadros abdominais agudos.

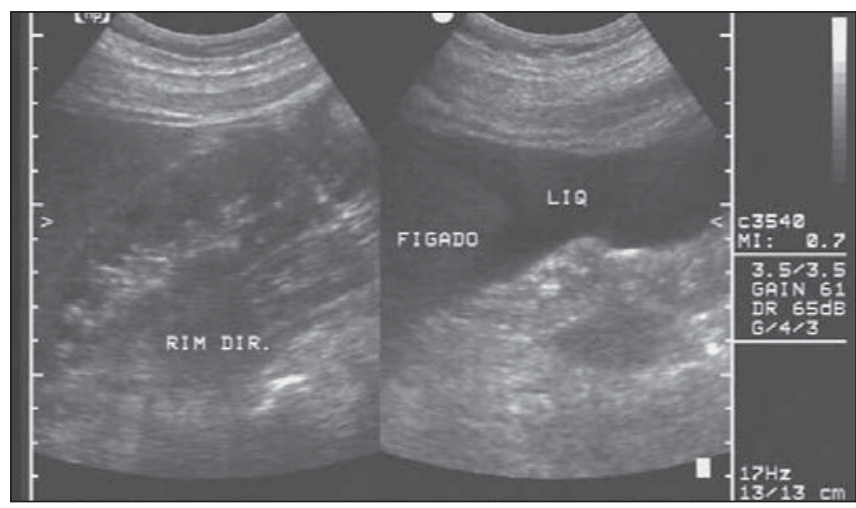

B

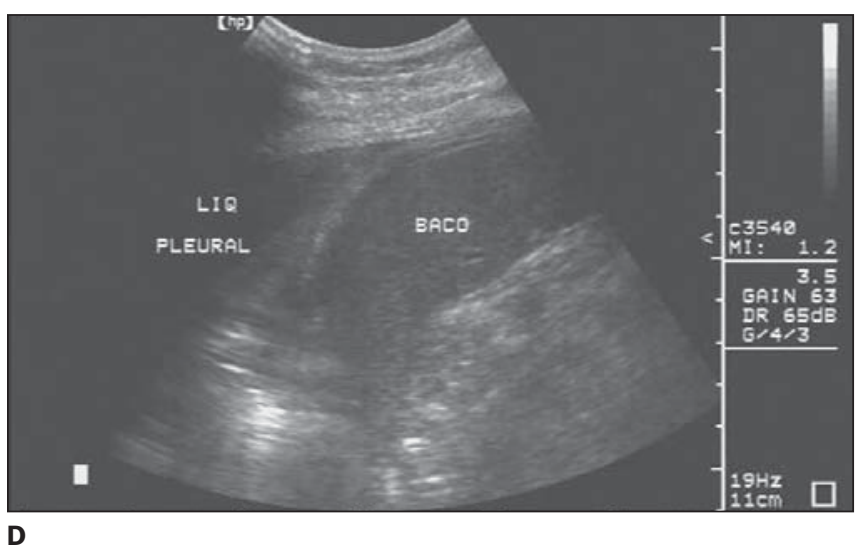

D

\section{REFERÊNCIAS}

1. Tauil PL. Urbanização e ecologia do dengue. Cad Saúde Pública 2001;17(suplemento):99-102.

2. Schatzmayr HG. Viroses emergentes e reemergentes. Cad Saúde Pública 2001;17(suplemento):20913.

3. Cortiñas MG, González DV, Cordero JC, Oliveras MLL. Dengue hemorrágico. Estudio clínico de 200 pacientes. Rev Cubana Med 1999;38:13-8.

4. Schatzmayr HG. Dengue situation in Brazil by year 2000. Mem Inst Oswaldo Cruz 2000;95(Suppl. I): 179-81.

5. Rocco IM, Kavakama BB, Santos CLS. First isolation of dengue 3 in Brazil from an imported case. Rev Inst Med Trop São Paulo 2001;43:55-7.

6. Nogueira RMR, Miagostovich MP, Filippis AMB, Pereira MAS, Schatzmayr HG. Dengue virus type 3 in Rio de Janeiro, Brazil. Mem Inst Oswaldo Cruz 2001;96:925-6.

7. Brasil. Ministério da Saúde. Fundação Nacional de Saúde. Dengue: aspectos epidemiológicos, diagnóstico e tratamento. Brasília, DF: Fundação Nacional de Saúde, 2002 (Série A. Normas e manuais técnicos no 176 ).

8. Setiawan MW, Samsi TK, Wulur H, Sugianto D, Pool TN. Dengue haemorrhagic fever: ultrasound as an aid to predict the severity of the disease. Pediatr Radiol 1998;28:1-4.

9. Nimmannitya S. Dengue haemorrhagic fever in
Thailand. (Abstract). Southeast Asian J Trop Med Public Health 1987;18:291-4.

10. Pramuljo HS, Harun SR. Ultrasound findings in dengue haemorrhagic fever. Pediatr Radiol 1991; 21:100-2.

11. Rumack CM, Wilson SR, Charboneau JW. Tratado de ultra-sonografia diagnóstica. $2^{2}$ ed. Rio de Janeiro, RJ: Guanabara Koogan, 1999:73-81, 131-7, $150-2$.

12. Bhamarapravati N, Tuchinda P, Boonyapaknavik V. Pathology of Thailand haemorrhagic fever: a study of 100 autopsy cases. Ann Trop Med Parasitol 1967;61:500-10.

13. Setiawan MW, Samsi TK, Pool TN, Sugianto D, Wulur H. Gallbladder wall thickening in dengue hemorrhagic fever: an ultrasonographic study. J Clin Ultrasound 1995;23:357-62.

14. Thulkar S, Sharma S, Srivastava DN, Sharma SK, Berry M, Pandey RM. Sonographic findings in grade III dengue haemorrhagic fever in adults. J Clin Ultrasound 2000;28:34-7.

15. Van Troys H, Gras C, Coton T, Deparis X, Tolou H, Durand JP. Dengue hémorragique d'importation: à propos d'un cas ayant présenté des signes de cholécystite ainguë alithiasique. Méd Trop 2000;60: 278-80.

16. Setiawan MW, Samsi TK, Wulur H, Sugianto D, Pool TN. Epigastric pain and sonographic assessment of the pancreas in dengue hemorrhagic fever. J Clin Ultrasound 1998;26:257-9. 